\title{
Adherence to guidelines for the prevention of HIV-related respira- tory diseases
}

\author{
J.S.G. Montaner*, P. Phillips**, C. Zala**, K.J.P. Craib*, \\ M.V. O'Shaughnessy*, M.T. Schechter ${ }^{+}$
}

Adherence to guidelines for the prevention of HIV-related respiratory diseases. J.S.G. Montaner, P. Phillips, C. Zala, K.J.P Craib, M.V. O'Shaughnessy, M.T. Schechter. (C)ERS Journals Ltd 1996.

ABSTRACT: In this study we characterized the pattern of use of preventive therapies for specific respiratory diseases within a cohort of homosexual men and assessed the impact of targeted feedback on the level of compliance with guidelines for these diseases.

All human immunodeficiency virus seronegative (HIV-) $(n=169)$ and acquired immune deficiency syndrome (AIDS)-free human immunodeficiency virus seropositive $(\mathrm{HIV}+)(\mathrm{n}=154)$ participants in our cohort, who completed four annual visits between October 1989 and December 1993, were identified. Information about the use of purified protein derivative (PPD) (tuberculin) testing, history of pneumococcal vaccinations, influenza vaccinations, use of Pneumocystis carinii pneumonia (PCP) prophylaxis, symptoms and CD4 counts was obtained yearly for each subject. In 1992, participating physicians were provided with feedback regarding the overall levels of compliance with contemporary guidelines for the prevention of respiratory disease. As part of this exercise, the guidelines were distributed and discussed.

The percentage of HIV+ patients who underwent PPD testing increased from 43 to $65 \%$ during the study ( $=0.001)$. Significantly more HIV+ than HIV-patients underwent PPD testing $(\mathrm{p}<0.001)$. A total of $144(94 \%) \mathrm{HIV}+$ men received at least one influenza vaccination compared to $60(35 \%)$ HIV- men $(p<0.001)$. Utilization of influenza vaccination in the HIV+ group significantly increased from $78 \%$ in 1992 to $92 \%$ in $1993(\mathrm{p}<0.001)$. A total of $104(68 \%)$ HIV + men received pneumococcal vaccination compared to $2(1 \%)$ HIV- men $(p<0.001)$. Among HIV+ individuals whose absolute CD4+ count was less than 200 cells $\cdot \mathrm{mm}^{-3}$, the percentage of men who received primary PCP prophylaxis was $0,86,72$ and 88 for the years 1990-1993, respectively. Among HIV+ patients whose only eligibility criterion for PCP prophylaxis was a CD4+ percentage $<20 \%$, compliance was $55,30,37$ and $50 \%$ for the years 1990-1993, respectively. Among HIV+ subjects, increases in the compliance level were noted for all preventive therapies after targeted feedback was provided during the last quarter of 1992. However, only utilization of influenza vaccine exceeded a $90 \%$ compliance in 1993.

These data demonstrate that a suboptimal level of compliance with current guidelines for the prevention of respiratory disease among human immunodeficiency virus-infected individuals can be significantly improved using targeted feedback. Although it is likely that similar effects could be achieved in other populations or the community at large, this remains to be demonstrated.

Eur Respir J., 1996, 9, 2318-2322.
*British Columbia Centre for Excellence in HIV/AIDS, **Division of Infectious Diseases, and Infectious Diseases Clinic, St Paul's Hospital, University of British Columbia, Vancouver, BC, Canada. ${ }^{+}$Dept of Health Care and Epidemiology, Faculty of Medicine, University of British Columbia, Vancouver, BC, Canada.

Correspondence: J.S.G. Montaner British Columbia Centre for Excellence in HIV/AIDS

St Paul's Hospital

University of British Colombia

Vancouver

$\mathrm{BC}$

Canada

\section{Keywords:}

Acquired immune deficiency syndrome

human immunodeficiency virus

Pneumocystis carinii

pneumonia

prophylaxis

tuberculosis

Received: November 101995

Accepted after revision May 151996

This work was supported by a grant from the National Health Research Development Program (NHRDP) of the Dept of National Health and Welfare of Canada. JSGM is a National Health Research Scholar and MTS is a National Health Research Scientist of the NHRDP.
Despite prompt diagnosis and appropriate therapy, the morbidity and mortality associated with preventable human immunodeficiency virus (HIV)-related infections remains high [1]. This provides a compelling rationale for the development of strategies to increase compliance with preventive therapies whenever possible. In this context, Pneumocystis carinii pneumonia (PCP) prophylaxis, tuberculin skin testing (purified protein derivative (PPD)), and vaccination both against influenza and pneumococcus have been widely recommended [2-7]. The safety and efficacy of primary and secondary PCP prophylaxis have been firmly established [8-10]. On the other hand, the clinical usefulness of PPD skin testing, influenza and pneumococcal vaccination in patients with HIV disease appears to vary, depending on a number of factors, such as the prevalence of tuberculosis or degree of immunodeficiency [11-15].

Current guidelines recommend that all HIV-infected individuals receive an annual tuberculin skin test, an annual influenza vaccine, and a single pneumococcal vaccine. Additionally, PCP prophylaxis was initially recommended for patients with an absolute CD4+ count of less than 200 cells $\cdot \mathrm{mm}^{-3}$ or a CD4+ percentage less than 20\% [2]. These guidelines were revised in August 1992; at that time, the CD4+ percentage criterion was deleted and unexplained fever (for $\geq 2$ weeks) or thrush, 
regardless of CD4+ cell count were added, as criteria for the initiation of PCP prophylaxis [3]. PCP prophylaxis is also recommended for all HIV-infected individuals who have had a prior episode of PCP $[2,3]$.

The present study was undertaken to characterize the pattern of use of preventive therapies for specific respiratory diseases within a cohort of homosexual men and to assess the impact of targeted feedback on the level of compliance with the aforementioned guidelines.

\section{Methods}

As described previously [16], the Vancouver lymphadenopathy AIDS study (VLAS) is an ongoing prospective study of 1,000 homosexual men recruited through eight general practices in central Vancouver. These practices are heavily involved in the care of HIV infected individuals. A total of 729 men were enrolled in the study between November 1982 and December 1984, and an additional 271 men were enrolled between October 1986 and December 1987. From November 1982 to September 1986, subjects returned for follow-up visits approximately every 6 months. Since October 1986, subjects have returned for follow-up visits on an annual basis. At each visit, subjects completed a detailed selfadministered questionnaire, underwent a complete physical examination, and had blood samples drawn for immunological and HIV-1 antibody testing.

For the purpose of this analysis, we identified all human immunodeficiency virus-seronegative (HIV-) and seropositive (HIV+) participants in the study who completed four annual visits between October 1989 and December 1993. The HIV+ group included subjects who were infected prior to October 1989 and were acquired immune deficiency syndrome (AIDS)-free on December 31, 1993. HIV-subjects served as a comparison group. Information regarding tuberculin skin testing, influenza and pneumococcal vaccinations, and use of PCP prophylaxis was obtained for each subject through his general practitioner at the time of each follow-up visit. Information about the use of influenza and pneumococcal vaccines was collected in October 1991 and thereafter. Data regarding CD4+ counts and clinical symptoms were used to stratify seropositive subjects and to assess compliance with the current recommendations for PCP prophylaxis. The 1992 revised guidelines for primary PCP prophylaxis omitted the CD4+ percentage of less than $20 \%$ as a threshold for initiating prophylaxis [3]. In view of this, the results were analysed for each of these two eligibility criteria separately.

Targeted feedback was specifically provided to participating physicians at group meetings held in late 1992, as part of the regular cohort meetings; once at medical rounds and once in writing through a letter to each physician participating in this study. Targeted feedback specifically dealt with compliance rates with the contemporary guidelines for the prevention of respiratory disease among HIV-infected individuals for the period 1990-1991, and aimed to review and discuss the guidelines with participating physicians.

Comparisons of serological groups with respect to preventive measures were conducted using Pearson's Chi-squared statistic. Mantel's trend test was used to investigate changes in utilization of preventive measures within each serological group, before and after targeted feedback was implemented.

\section{Results}

Of the 1,000 participants who enrolled in the VLAS, 14 had unknown serological status, 254 had developed AIDS, 279 moved away or withdrew from the study prior to October 1989, and 121 completed less than four visits during the period October 1989 to December 1993. A total of 323 patients (154 HIV+ and 169 HIV- men) and eight physicians were included in this analysis. The number of patients per physician ranged from $13(4 \%)$ to $88(28 \%)$. The median time elapsed between successive visits for all subjects was 12 months, and was similar in both serological groups. With regard to demographic characteristics, HIV+ men were significantly younger than HIV-men (median age 36 vs 39 yrs; $p=0.028$ ), and were less likely to have attended university (63 vs 80\%; $\mathrm{p}=0.001)$. Similar percentages of HIV+ and HIV- men reported having annual incomes greater than $\$ 10,000$ (86 vs $87 \%$; $\mathrm{p}=0.915)$.

\section{Tuberculin skin testing}

Table 1 shows the frequency of tuberculin skin testing in both serological groups. The percentage of HIV+ patients who underwent TB skin testing increased significantly from 43 to $65 \%$ during the observation period $(\mathrm{p}=0.001)$. Significantly more HIV+ patients underwent skin testing at all four visits than did HIV- patients $(\mathrm{p}<0.001)$. A total of $119(77 \%) \mathrm{HIV}+$ patients were tested at least once during the observation period, compared to $83(49 \%)$ in the HIV- group $(\mathrm{p}<0.001)$. A significant increase in skin test utilization was also noted among HIV- patients. During the observation period, the percentage of seronegative men who underwent testing increased from 12\% in 1990 to $40 \%$ in 1993 ( $\mathrm{p}=0.001$ ). We speculate that the increase in tuberculin testing of HIV- individuals represents a nonspecific effect of the targeted feedback.

Table 1. - Utilization of tuberculin skin testing, influenza and pneumococcal vaccines in 154 HIV+ and 169 HIVmen at four annual visits (October 1989 until December 1993)

\begin{tabular}{lcccccrrr}
\hline Preventative therapy & \multicolumn{2}{c}{$1989-1990$} & \multicolumn{2}{c}{$1990-1991$} & \multicolumn{2}{c}{$1991-1992$} & \multicolumn{2}{c}{$1992-1993$} \\
& HIV & HIV- & \multicolumn{1}{c}{ HIV +} & HIV- & \multicolumn{1}{c}{ HIV+ } & HIV- & HIV+ & HIV- \\
\hline Tuberculin skin test & 66 $(43)$ & $21(12)$ & 69 $(45)$ & $21(12)$ & $78(51)$ & $100(65)$ & $100(65)$ & $67(40)$ \\
Influenza vaccine & NA & NA & NA & NA & $120(78)$ & $45(27)$ & $141(92)$ & $51(30)$ \\
Pneumococcal vaccine & NA & NA & NA & NA & $36(23)$ & $1(<1)$ & $103(67)$ & $2(1)$ \\
\hline
\end{tabular}

Absolute values are presented, and percentage in parenthesis. HIV+: human immunodeficiency virus seropositive; HIV-: human immunodeficiency virus seronegative; NA: not available. 


\section{Influenza vaccinations}

A total of $144(94 \%) \mathrm{HIV}+$ men received at least one vaccination during the 2 year period compared to 60 $(35 \%)$ of HIV- men $(\mathrm{p}<0.001)$. As seen in table 1 , at both visits, significantly more HIV+ men received an annual influenza vaccination than HIV- men $(\mathrm{p}<0.001)$. Utilization of influenza vaccine in the HIV+ group significantly increased from $78 \%$ (120 out of 154) in 1992 to $92 \%$ (141 out of 154$)$ in $1993(\mathrm{p}<0.001)$. The percentage of HIV+ men who received an influenza vaccine did not change significantly from 1992 to 1993 (27 vs $30 \%$; $\mathrm{p}=0.470$ ).

\section{Pneumococcal vaccinations}

A total of $104(68 \%)$ HIV+ men received pneumococcal vaccination during this 2 year period compared to $2(1 \%)$ of HIV- men $(\mathrm{p}<0.001)$. As expected, significantly more HIV+ men received pneumococcal vaccination than HIV- men at both visits $(\mathrm{p}<0.001)$. Utilization of pneumococcal vaccine in the HIV+ group significantly increased from 23\% (36 out of 154) in 1992 to $67 \%$ (103 out of 154$)$ during $1993(\mathrm{p}<0.001)$.

\section{PCP prophylaxis}

The HIV+ group was classified according to absolute CD4+ count, CD4+ percentage and symptoms at each visit. During the observation period, the number of participants who were eligible for PCP prophylaxis significantly increased. For example, the percentage of men with CD4+ <200 cells $\cdot \mathrm{mm}^{-3}$ increased from 3\% (4 out of 154) in 1990 to $22 \%$ (33 out of 152) in 1993 (table 2).

Among individuals whose absolute CD4+ count was $<200$ cells $\cdot \mathrm{mm}^{-3}$, the percentage of men who received primary PCP prophylaxis was $0 \%$ ( 0 out of 4$), 86 \%$ (6 out of 7 ), $72 \%$ (21 out of 29), and $88 \%$ (29 out of 33) for the years 1990-1993, respectively. Among those whose CD4+ percentage was less than $20 \%$, the percentage of men who were taking primary $\mathrm{PCP}$ prophylaxis was $44 \%$ (7 out of 16), $38 \%$ (12 out of 32 ), $52 \%$ (36 out of 69 ) and $63 \%$ (52 out of 82 ), respectively. It should be noted that patients with either one of these eligibility criteria may have had additional concomitant eligibility criteria (i.e. not mutually exclusive).

Finally, compliance with individual eligibility criteria for PCP prophylaxis was examined by restricting the

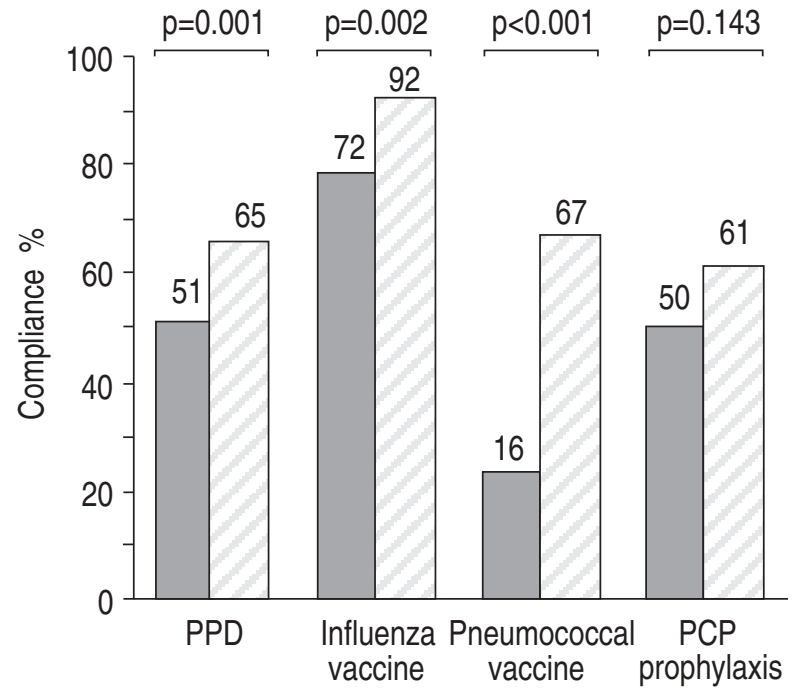

Fig. 1. - Level of compliance with specific preventative interventions before (1992) and after (1993) targeted feedback. $\square$ 1992;

$\square$ : 1993. PPD: purified protein derivative; PCP: Pneumocystis carinii pneumonia.

analysis to those individuals who met only one eligibility criterion. Almost all patients who had an absolute CD4+ count $<200$ cells $\cdot \mathrm{mm}^{-3}$, also had concomitant eligibility criteria. Consequently, this analysis for individual criteria was only available for; 1) CD4+ percentage $<20 \%$; and 2) the presence of thrush or unexplained fever. It should be noted that although thrush or unexplained fever ( $\geq 2$ weeks) have been identified as independent predictors of PCP in the 1989 Centres for Disease Control (CDC) guidelines, at that time they were not listed as specific indications for primary prophylaxis, regardless of CD4+ counts. Therefore, fever or thrush as eligibility criteria for primary PCP prophylaxis only applied to the final 17 months of the observation period (August 1992 until December 1993). Among patients whose only eligibility criterion was CD4+ percentage $<20 \%$, compliance was $55 \%$ ( 0 out of 11 ), $30 \%$ (7 out of 23 ), 37\% (13 out of 35) and 50\% (18 out of 36) for the years 1990-1993, respectively. Among patients whose only eligibility criterion was thrush and/or unexplained fever, the compliance rates were $0 \%$ ( 0 out of 7 ), $29 \%$ (4 out of 14 ), $0 \%$ (0 out of 4 ) and $20 \%$ ( 1 out of 5) for the years 1990 to 1993 , respectively. Of note, thrush and/or unexplained fever were infrequently encountered in the absence of other criteria for the initiation of PCP prophylaxis in our cohort.

Table 2. - Compliance with PCP prophylaxis among HIV+ men during four annual visits according to eligibilty criteria (1990-1993)*

\begin{tabular}{lcrrr}
\hline Variable & 1990 & 1991 & 1992 & 1993 \\
\hline CD4+ count $\leq 200$ & $0 / 4(0)$ & $6 / 7(86)$ & $21 / 29(72)$ & $29 / 33(88)$ \\
CD4 $\% \leq 20 \%$ & $7 / 16(44)$ & $12 / 32(38)$ & $36 / 69(52)$ & $52 / 82(63)$ \\
CD4+ count $\leq 200$ or & $7 / 17(41)$ & $14 / 34(41)$ & $38 / 72(53)$ & $53 / 83(64)$ \\
CD4+ \% $\leq 20 \%$ & & & & \\
Fever or thrush & $1 / 9(11)$ & $5 / 18(28)$ & $11 / 22(50)$ & $14 / 28(50)$ \\
Any of the above & $7 / 24(29)$ & $18 / 48(31)$ & $38 / 76(50)$ & $54 / 88(61)$ \\
\hline
\end{tabular}

*: PCP prophylactics included any of the following regimens, dapsone, pentamidine or septra. Values are presented as the number of subjects compliant with PCP prophylaxis/number of subjects meeting criterion for prophylaxis, and percentage compliance in parenthesis. PCP: Pneumocystis carinii pneumonia; HIV+: human immunodeficiency virus seropositive. 


\section{The effect of targeted feedback}

Figure 1 summarizes the effect of targeted feedback on the level of compliance with contemporary guidelines. Among HIV+ subjects, increases in the compliance level were noted for all preventive therapies after targeted feedback was provided in 1992. However, only utilization of influenza vaccine exceeded a $90 \%$ compliance in 1993.

\section{Discussion}

The present data demonstrate that PPD testing, PCP prophylaxis, and influenza and pneumococcal vaccines are used more frequently among HIV+ than HIV- homosexual males. Despite this, the results indicate that levels of compliance are suboptimal, in the $23-78 \%$ range. However, it has also been demonstrated that compliance can be dramatically increased to the 65-92\% range with the use of targeted feedback.

This survey was limited to a well-characterized cohort of homosexual men who are highly educated in HIV/AIDS-related issues. Also, this cohort is being medically followed through a limited number of general practitioners with a high level of interest and expertise in the field of HIV/AIDS management. Consequently, we anticipate that the levels of compliance within our cohort are likely to be higher than those that could be found among HIV-infected individuals in the community.

These results expand those recently published by GLASSROTh et al. [17], who studied 1,171 AIDS-free, HIV-infected individuals throughout the United States. Although PCP prophylaxis use was high in their cohort, pneumococcal vaccine and tuberculin skin testing were used by less than $30 \%$ of those eligible. The fact that we were able to significantly improve compliance with current guidelines through the use of targeted feedback suggests that similar approaches could be used on a wider scale to enhance the management of HIV/AIDS in the community setting. Although the temporal relationship between targeted feedback and increased compliance described here is suggestive of a causal association, this study does not exclude the possible contribution of progression of knowledge over the study period.

A number of issues could contribute to poor compliance with therapeutic guidelines. Among them, lack of awareness, poor acceptance by physicians and/or patients, ease of access, cost and safety profile of the various preventive measures could play a significant role in this respect. Of note, it has been demonstrated that effectiveness of these interventions may decrease with time or as HIV disease progresses [5, 14, 18]. Whatever the mechanism leading to the suboptimal use of the specific preventive therapies under study, the present data suggest that this can be partially reversed with the use of targeted feedback. This leads us to believe that physician awareness and attitude towards such interventions play a significant role in determining the level of compliance. These data, however, offer no insight regarding the role of patient-related issues in this context.

Recently, WORTLEY et al. [15] reported on the pneumococcal and influenza vaccination levels among HIVinfected adolescents and adults in the United States. This medical record review involved more than 90 clinics, hospitals and private medical practices in nine cities in the USA over a 12 month period. Overall, 3,570 of 9,796 (37\%) and 2,047 of 6,161 (33\%) HIV-infected persons had received pneumococcal and influenza vaccine, respectively. Higher rates of compliance were reported by BROISMAN [18] in a specialized HIV clinic. In this report, 92, 90 and $96 \%$ compliance was reported for tuberculin skin test, influenza and pneumococcal vaccine, respectively. In the same study, lower rates of compliance were seen among $78 \mathrm{HIV}$-infected individuals followed in a general internal medicine clinic. In this group, the compliance was reported to be 74,53 and $56 \%$ for tuberculin skin testing, influenza and pneumococcal vaccine, respectively $(\mathrm{p}=0.001)$. Compliance with guideline for PCP prophylaxis was reported to be $100 \%$ both in the specialized and the internal medicine clinic. Of note, the report by BROISMAN [18] was limited to an intention to treat analysis, failing to provide the true level of compliance in the study population. Despite this, the above data, in combination with our own results, suggest that the level of compliance with therapeutic guidelines increases with the level of awareness within the care setting. This lends support to the notion that specialized clinics devoted to health promotion and preventive interventions can substantially increase the level of compliance with these guidelines, as proposed by BELCHER [19] and GLASSROTH et al. [17].

It should be stressed that despite multiple publications supporting the use of current guidelines, the supportive evidence is not uniform [5, 14, 20-26]. In contrast to the unequivocal evidence in favour of primary and secondary PCP prophylaxis [8-10], the usefulness of repeated PPD skin testing, influenza and pneumococcal vaccination has been less thoroughly documented [11-15]. Furthermore, a number of studies have identified limitations of the latter interventions within specific subgroups of HIV-infected patients. For example, HIV-infected patients with low CD4+ counts have been shown to generate suboptimal antibody responses both to influenza and pneumococcal vaccines, which may not be clinically protective $[14,15]$. In addition, the results of PPD skin testing are at least partly confounded by HIV-related anergy, the frequency of which is inversely related to the CD4+ count [11-13, 17]. With regard to the low level of PPD use in our cohort, it is also important to emphasize that despite the present study being restricted to AIDS-free individuals, PPD reactivity is known to be relatively low in our cohort [16]. Furthermore, available data suggest that Mycobacterium tuberculosis was relatively infrequent among HIV-infected individuals in our area at the time of this study [27].

Of note, a recent policy analysis by Rose et al. [13] suggests that pneumococcal vaccination of HIV-infected individuals remains a highly cost-effective intervention. In their study, they estimated that 17 and 94 individuals would need to be vaccinated in order to prevent a single episode of pneumococcal disease and a single pneumococcal disease-related death, respectively. They also estimated that each pneumococcal vaccination would be associated with an overall average extension in life expectancy of 14 days per vaccinated patient and a net cost-saving to the community of $\$ 187$. In the same analysis, 4,000 and 45,000 influenza vaccinations were required 
to prevent one influenza-related hospitalization and 12 influenza related-deaths. The average extension of life expectancy per patient vaccinated was $10 \mathrm{~h}$ and the cost of the vaccination to save a single life was $\$ 84,000$. Against this, in the present study, the highest baseline compliance rate was originally found for influenza vaccine, for which the rationale as well as the cost-effectiveness appears to be least compelling. Once again the use of targeted feedback allowed us to improve this situation.

In summary, these data demonstrate that a suboptimal level of compliance with current guidelines for the prevention of respiratory disease among human immunodeficiency virus-infected individuals can be substantially improved using targeted feedback. The results pertain to a well-characterized cohort of homosexual men. Although it is likely that similar effects could be achieved in other populations or the community at large, this remains to be demonstrated.

Acknowledgements: This work could not have been possible without the commitment and support of the participating volunteers. The authors are indebted to S. Broughton, B. Douglas, M. Maynard, R. Nitz, P. Sestak, R. Voigt, B. Willoughby, B. Woodfall, S. Fay for their clinical support. They also thank B. Devlin for her assistance and D. Hamann-Trou and K. Hsu for secretarial assistance.

\section{References}

1. Montaner JSG, Le TN, Hogg RS, et al. The changing spectrum of AIDS index diseases in Canada. AIDS 1994; 8: 693-696.

2. Centers for Disease Control. Guidelines for prophylaxis against Pneumocystis carinii pneumonia for persons infected with human immunodeficiency virus. $M M W R$ 1989; 38: 1-9.

3. Centers for Disease Control. Recommendations for prophylaxis against Pneumocystis carinii pneumonia for adults and adolescents infected with human immunodeficiency virus. MMWR 1992; 41 (RR-4): 1-11.

4. Centers for Disease Control. Tuberculosis and human immunodeficiency virus infection: recommendations of the Advisory Committee for the elimination of tuberculosis. MMWR 1989; 38: 236-250.

5. Centers for Disease Control. Purified protein derivative (PPD), tuberculin, anergy and HIV infection: guidelines for anergy testing and management of anergic persons at risk of tuberculosis. MMWR 1991; 40 (RR-5): 27-33.

6. Centers for Disease Control. Prevention and control of influenza: recommendations of Immunization Practices Advisory Committee (ACIP). MMWR 1991; 40: 1-15.

7. Centers for Disease Control. Pneumococcal recommendations of the Immunization Practices Advisory Committees: pneumococcal polysaccharide vaccine. MMWR 1989; 38: 64-76.

8. Leoung GS, Feigal DW, Montgomery AB, et al. Aerosolized pentamidine for prophylaxis against Pneumocystis carinii pneumonia. N Engl J Med 1990; 323: 769-775.

9. Montaner JSG, Lawson LM, Gervais A, et al. A placebo-controlled study of aerosol pentamidine for the secondary prophylaxis of AIDS-related PCP. Ann Intern Med 1991; 114(11): 948-953.

10. Hirschel B, Lazzarin A, Chopard P, et al. A controlled study of inhaled pentamidine for primary prevention of Pneumocystis carinii pneumonia. N Engl J Med 1991; 334: 1079-1083.

11. Shapiro ED, Berg AT, Austrian R, et al. The protective efficacy of polyvalent pneumococcal polysaccharide vaccine. N Engl J Med 1991; 325: 1453-1460.

12. Broome CV, Breiman RS. Pneumococcal vaccine test present and future. N Engl J Med 1991; 325: 1506-1508.

13. Rose BN, Schechter CB, Sacks HS. Influenza and pneumococcal vaccination of HIV-infected patients: a policy analysis. Am J Med 1993; 94: 160-168.

14. Nelson KE, Clements LM, Miotti P, Cohn S, Polk BF. The influence of human immunodeficiency virus (HIV) infection on antibody responses to influenza vaccine. Ann Intern Med 1988; 109: 383-388.

15. Wortley P, Farizo K, Chu F. Influenza and pneumococcal vaccination coverage among patients with HIV infection in the United States. IXth International Conference on AIDS, Berlin 1993, Vol. I. Abstract Po-B32-2249: 510.

16. Marion SA, Schechter MT, Weaver MS, et al. Evidence that prior immune dysfunction predisposes to human immunodeficiency virus Infection in homosexual men. J Acquir Immune Defic Syndr 1989; 2: 178-186.

17. Glassroth J, Jordan M, Wallace J, et al. Use of preventive interventions by persons infected with type-1 human immunodeficiency virus (HIV-1). Am J Prev Med 1994; 10: 259-266.

18. Broisman L. A comparison of two systems of delivery of care to HIV-infected patients. IXth International Conference on AIDS, Berlin 1993, Vol. 1. Abstract PoB32-2254: 511.

19. Belcher DW. Implementing preventive services: success and failure in an out-patient trial. Arch Intern Med 1990; 150: 2533-2541.

20. Centers for Disease Control. Screening for tuberculosis and tuberculosis infection in high-risk populations: recommendations of the Advisory Committee for Elimination of Tuberculosis. MMWR 1990; 39(RR-8): 1-7.

21. Gardner P, Schaffner W. Immunization of adults. N Engl J Med 1993; 328: 1252-1258.

22. American College of Physicians Task Force on Adult Immunization, Infectious Diseases Society of American Guide for Adult Immunizations. Second Edn. Philadelphia; American College of Physicians, 1990.

23. Fedson DS, Harward MP, Reid RA, Kaiser DL. Hospitalbased pneumococcal immunization: epidemiologic rationale from the Shenandoah study. JAMA 1990; 264: $1117-1122$

24. Safran S, Rush GD, Mills J. Influenza in patients with human immunodeficiency virus infection. Chest 1990; 98: 33-37.

25. Girard PMG, Landman R, Gaudebout C, et al. Dapsonepyrimethamine compared with aerosol pentamidine as primary prophylaxis against Pneumocystis carinii pneumonia and toxoplasmosis in HIV infection. $N$ Engl $J$ Med 1993; 328: 1514-1520.

26. Kroon FP, van Dissel JT, de Jong JC, van Furth R. Antibody response to influenza, tetanus and pneumococcal vaccines in HIV-seropositives individuals in relation to the number of CD4+ lymphocytes. AIDS 1994, 8: 469-476.

27. Korzeniewska-Kosela M, Fitzgerald M, Vedal S, Allen EA, et al. Spectrum of tuberculosis with HIV infection in British Columbia: report of 40 cases. Can Med Assoc J 1992; 146(11): 1927-1934. 